PHYSICAL REVIEW D 93, 054017 (2016)

\title{
Gauge-invariant field-strength correlators for QCD in a magnetic background
}

\author{
Massimo D’Elia, ${ }^{*}$ Enrico Meggiolaro, ${ }^{\dagger}$ Michele Mesiti, ${ }^{\ddagger}$ and Francesco Negro ${ }^{\S}$ \\ Dipartimento di Fisica dell'Università di Pisa and INFN, \\ Sezione di Pisa, Largo Pontecorvo 3, I-56127 Pisa, Italy \\ (Received 12 November 2015; published 9 March 2016)
}

\begin{abstract}
We consider the properties of the gauge-invariant two-point correlation functions of the gauge-field strengths for QCD in the presence of a magnetic background field at zero temperature. We discuss the general structure of the correlators in this case and provide the results of an exploratory lattice study for $N_{f}=2$ QCD discretized with unimproved staggered fermions. Our analysis provides evidence for the emergence of anisotropies in the nonperturbative part of the correlators and for an increase of the gluon condensate as a function of the external magnetic field.
\end{abstract}

DOI: $10.1103 /$ PhysRevD.93.054017

\section{INTRODUCTION}

The study of strong interactions in the presence of strong magnetic fields has attracted increased interest in the last few years (see, e.g., Ref. [1]). This is justified by the great phenomenological relevance of the issue: the physics of some compact astrophysical objects, like magnetars [2], of noncentral heavy-ion collisions [3-7] and of the early Universe $[8,9]$ involve the properties of quarks and gluons in the presence of magnetic backgrounds going from $10^{10}$ Tesla up to $10^{16}$ Tesla $\left(|e| B \sim 1 \mathrm{GeV}^{2}\right)$. The study is also interesting from a purely theoretical point of view, since it reveals new nonperturbative features of non-Abelian gauge theories.

One emerging feature is that gluon fields, even if not directly coupled to electromagnetic fields, can be significantly affected by them. This is not unexpected, since effective QED-QCD interactions, induced by quark-loop contributions, can be important, because of the nonperturbative nature of the theory [10-26]. In particular, a uniform magnetic background is expected to lead to gluon-field anisotropies [10,11,16,22], which may also have phenomenological implications. This has been confirmed by various lattice QCD studies [27-34]. In particular, anisotropies have been observed in various pure-gauge quantities, like the average plaquettes taken in different planes [30,31] and the static quark-antiquark potential [35], with possible effects on the spectra of heavy quark bound states [36].

In the present study, looking for a more systematic analysis of the nonperturbative vacuum modifications induced by the magnetic field, we consider the gaugeinvariant two-point field-strength correlators (see Ref. [37] for a complete review on this subject), which are defined as

\footnotetext{
*massimo.delia@unipi.it †enrico.meggiolaro@unipi.it mesiti@pi.infn.it

${ }^{\S}$ fnegro@pi.infn.it
}

$$
\mathcal{D}_{\mu \rho, \nu \sigma}(x)=g^{2}\left\langle\operatorname{Tr}\left[G_{\mu \rho}(0) S(0, x) G_{\nu \sigma}(x) S^{\dagger}(0, x)\right]\right\rangle,
$$

where $G_{\mu \rho}=T^{a} G_{\mu \rho}^{a}$ is the field-strength tensor, $T^{a}$ are the $S U(3)$ generators in the fundamental representation, and $S(0, x)$ is the parallel transport from 0 to $x$ along a straight line (Schwinger line), which is needed to make the correlator gauge invariant. Such correlators have been first considered, together with analogous correlators involving fermionic fields, to take into account the nonuniform distributions of the vacuum condensates [38-41]. Then, they have been widely used to parametrize the nonperturbative properties of the QCD vacuum, especially within the framework of the so-called stochastic vacuum model [42-44], since they represent the leading (Gaussian) term in the cumulant expansion. Gluon-field correlators have been also exploited as a tool to explore the response of the QCD vacuum to external magnetic fields $[25,45]$; however this has usually been done by considering the unmodified correlators, i.e. computed at zero magnetic field. The question that we approach here is different: how are the zero-temperature correlators themselves modified by the background field?

A first issue to be considered regards the symmetry properties and the associated parametrization of the correlators. In the vacuum and in the absence of external sources, Lorentz symmetry implies a simple form for the two-point functions in Eq. (1), which can be expressed in terms of two independent scalar functions of $x^{2}$, which are usually denoted by $\mathcal{D}\left(x^{2}\right)$ and $\mathcal{D}_{1}\left(x^{2}\right)$ [42-44]:

$$
\begin{aligned}
\mathcal{D}_{\mu \rho, \nu \sigma}(x)= & \left(\delta_{\mu \nu} \delta_{\rho \sigma}-\delta_{\mu \sigma} \delta_{\rho \nu}\right)\left[\mathcal{D}\left(x^{2}\right)+\mathcal{D}_{1}\left(x^{2}\right)\right] \\
& +\left(x_{\mu} x_{\nu} \delta_{\rho \sigma}-x_{\mu} x_{\sigma} \delta_{\rho \nu}+x_{\rho} x_{\sigma} \delta_{\mu \nu}-x_{\rho} x_{\nu} \delta_{\mu \sigma}\right) \\
& \times \frac{\partial \mathcal{D}_{1}\left(x^{2}\right)}{\partial x^{2}}
\end{aligned}
$$

The presence of a uniform external field breaks Lorentz symmetry explicitly, so that such a parametrization is not 
justified any more, especially in light of the already observed propagation of symmetry breaking from the electromagnetic to the gluon sector. Therefore in Sec. II, on the basis of the residual symmetries of the theory, we discuss what form the correlators can take in this case.

Then, in Sec. III, we present an exploratory lattice determination of the gluon-field correlators in a magnetic field which is performed for $N_{f}=2 \mathrm{QCD}$, discretized by means of unimproved staggered fermions, and exploits cooling as a technique to smooth out ultraviolet fluctuations. Numerical results are analyzed, within the parametrization proposed in Sec. II, in order to clarify which properties of the gluon correlators are mostly affected by the presence of the magnetic field. The analysis then focuses on a quantity of phenomenological interest which can be extracted from the gluon correlators, the so-called gluon condensate. Finally, in Sec. IV, we draw our conclusions.

\section{FIELD CORRELATORS IN A CONSTANT MAGNETIC BACKGROUND}

As we have already emphasized in the introduction, the presence of an external field breaks Lorentz symmetry [SO (4) symmetry in the Euclidean space], so that the most general parametrization is more complex than the one reported in Eq. (2). Let us discuss this point in more detail.

The required general symmetry properties for the correlator in Eq. (1) are that it be left unchanged under exchange of the $\mu \rho$ and $\nu \sigma$ pairs, and antisymmetric both in the $\mu \rho$ and in the $\nu \sigma$ indices, i.e. we can write in general

$$
\mathcal{D}_{\mu \rho, \nu \sigma}=\sum_{n} f_{n} T_{\mu \rho, \nu \sigma}^{(n)},
$$

where (i) $T_{\nu \sigma, \mu \rho}^{(n)}=T_{\mu \rho, \nu \sigma}^{(n)}$, and (ii) $T_{\rho \mu, \nu \sigma}^{(n)}=T_{\mu \rho, \sigma \nu}^{(n)}=-T_{\mu \rho, \nu \sigma}^{(n)}$.

A class of tensors satisfying such properties is written as

$$
T_{\mu \rho, \nu \sigma}^{(A, B)} \equiv A_{\mu \nu} B_{\rho \sigma}-A_{\rho \nu} B_{\mu \sigma}-A_{\mu \sigma} B_{\rho \nu}+A_{\rho \sigma} B_{\mu \nu}
$$

where $A_{\mu \nu}$ and $B_{\mu \nu}$ are two rank-2 tensors which are both symmetric $\left(A_{\nu \mu}=A_{\mu \nu}, B_{\nu \mu}=B_{\mu \nu}\right)$ or both antisymmetric $\left(A_{\nu \mu}=-A_{\mu \nu}, B_{\nu \mu}=-B_{\mu \nu}\right)$.

In the absence of external background fields, there are only two available rank-2 tensors, which are symmetric and can be constructed in terms of the metric tensor $g_{\mu \nu}=\delta_{\mu \nu}$ and of the four-vector $x_{\mu}$ : they are $\delta_{\mu \nu}$ itself and $x_{\mu} x_{\nu}$, so that the most general parametrization reads as in Eq. (2),

$$
\mathcal{D}_{\mu \rho, \nu \sigma}=f_{1} T_{\mu \rho, \nu \sigma}^{(1)}+f_{2} T_{\mu \rho, \nu \sigma}^{(2)},
$$

where
$T_{\mu \rho, \nu \sigma}^{(1)} \equiv \frac{1}{2} T_{\mu \rho, \nu \sigma}^{(\delta, \delta)}=\delta_{\mu \nu} \delta_{\rho \sigma}-\delta_{\mu \sigma} \delta_{\rho \nu}$,

$T_{\mu \rho, \nu \sigma}^{(2)} \equiv T_{\mu \rho, \nu \sigma}^{(x x, \delta)}=x_{\mu} x_{\nu} \delta_{\rho \sigma}-x_{\mu} x_{\sigma} \delta_{\rho \nu}+x_{\rho} x_{\sigma} \delta_{\mu \nu}-x_{\rho} x_{\nu} \delta_{\mu \sigma}$,

$\left(T^{(x x, x x)}=0\right.$ by construction), while $f_{1} \equiv \mathcal{D}+\mathcal{D}_{1}$ and $f_{2} \equiv \frac{\partial \mathcal{D}_{1}}{\partial x^{2}}$ (or, equivalently, $\mathcal{D}$ and $\mathcal{D}_{1}$ ) are two scalar functions of $x^{2}$.

In the presence of an external background field $F_{\mu \nu}$, instead, many additional rank-2 tensors appear, both antisymmetric (like $F_{\mu \nu}$ itself or $H_{\mu \nu} \equiv h_{\mu} x_{\nu}-h_{\nu} x_{\mu}$, where $h_{\mu} \equiv F_{\mu \nu} x_{\nu}$ ) and symmetric (like $F_{\mu \nu}^{(2)} \equiv F_{\mu \alpha} F_{\alpha \nu}$ or $M_{\mu \nu} \equiv$ $p_{\mu} x_{\nu}+p_{\nu} x_{\mu}$, where $\left.p_{\mu} \equiv F_{\mu \nu}^{(2)} x_{\nu}=F_{\mu \alpha} h_{\alpha}\right)$. Correspondingly, many more terms appear in the parametrization in Eq. (3), with new rank-4 tensors like $\frac{1}{2} T_{\mu \rho, \nu \sigma}^{(F, F)}, T^{(F, H)}$, $T^{\left(\delta, F^{(2)}\right)}, T^{\left(x x, F^{(2)}\right)}, T^{(\delta, h h)}, T^{(\delta, M)}$ and so on. Moreover, for a magnetic field directed along the $z$ axis, the coefficients $f_{n}$ will depend separately on $x^{2}+y^{2}$ and on $z^{2}+t^{2}$, because of the breaking of the Euclidean $S O(4)$ symmetry. All that makes a numerical analysis based on the more general parametrization (3) of the correlator quite involved and not easily affordable.

On the other hand, in our present investigation on the lattice, we consider only correlators of the kind

$$
\mathcal{D}_{\mu \nu, \xi}(d) \equiv \mathcal{D}_{\mu \nu, \mu \nu}(x=d \hat{\xi})
$$

where the two plaquettes are parallel to each other and the separation $x$ is along one $(\hat{\xi})$ of the four basis vectors of the lattice $[\hat{x}=(1,0,0,0), \hat{y}=(0,1,0,0), \hat{z}=(0,0,1,0)$, $\hat{t}=(0,0,0,1)]$. These amount, in general, to 24 different correlation functions. Without any additional external field, the symmetries of the system group these 24 correlators into two equivalence classes, usually denoted (as in [46]) $\mathcal{D}_{\|}$(when $\xi=\mu$ or $\xi=\nu$ ) and $\mathcal{D}_{\perp}$ (when $\xi \neq \mu$ and $\xi \neq \nu$ ), with

$$
\begin{aligned}
\mathcal{D}_{\|} & =\mathcal{D}+\mathcal{D}_{1}+x^{2} \frac{\partial \mathcal{D}_{1}}{\partial x^{2}}, \\
\mathcal{D}_{\perp} & =\mathcal{D}+\mathcal{D}_{1}
\end{aligned}
$$

The dependence of the correlators on the distance $d$, in the case of zero external field, has been directly determined by numerical simulations on the lattice in $[46-50]^{1}$ : the functions $\mathcal{D}$ and $\mathcal{D}_{1}$ have been parametrized in the form

\footnotetext{
${ }^{1}$ In another approach [51], the correlators have been extracted from lattice calculations of the heavy-quark potential, by analyzing field-strength insertions into a Wilson loop under the assumption of factorization, as in the stochastic vacuum model.
} 


$$
\begin{gathered}
\mathcal{D}=\frac{a_{0}}{d^{4}}+A_{0} e^{-\mu d}, \\
\mathcal{D}_{1}=\frac{a_{1}}{d^{4}}+A_{1} e^{-\mu d},
\end{gathered}
$$

where the terms $\sim 1 / d^{4}$ are of perturbative origin and are necessary to describe the short-distance behavior of the correlators, while the exponential terms represent the nonperturbative contributions. In particular, the coefficients $A_{0}$ and $A_{1}$ can be directly linked to the gluon condensate of the QCD vacuum [see Eq. (20) below], while the correlation length $1 / \mu$, which sets the scale of the spatial variations of the nonperturbative vacuum fluctuations, governs the effect of the condensate on the levels of $Q \bar{Q}$ bound states [38-41], and, moreover, enters the description of various QCD vacuum models [42-44]. In Refs. [47,48], the perturbativelike terms had the form $\sim e^{-\mu_{a} d} / d^{4}$ : in the present work, instead (following what was done also in Ref. [50]), we have neglected the exponential term $e^{-\mu_{a} d}$, by fixing $\mu_{a}=0$, since, in the spirit of the operator product expansion, we concentrate on the behavior of the correlators at short distances. In terms of the quantities $\mathcal{D}_{\|}$and $\mathcal{D}_{\perp}$ defined in Eq. (8), the parametrization (9) reads

$$
\begin{aligned}
& \mathcal{D}_{\|}=\left[A_{0}+A_{1}\left(1-\frac{1}{2} \mu d\right)\right] e^{-\mu d}+\frac{a_{0}-a_{1}}{d^{4}}, \\
& \mathcal{D}_{\perp}=\left(A_{0}+A_{1}\right) e^{-\mu d}+\frac{a_{0}+a_{1}}{d^{4}} .
\end{aligned}
$$

In the presence of a constant and uniform magnetic field $\vec{B}$ oriented along the $z$ axis, i.e. $F_{x y} \neq 0$, the $S O(4)$ Euclidean symmetry breaks into

$$
S O(2)_{x y} \otimes S O(2)_{z t}
$$

By virtue of this residual symmetry [which implies two equivalence relations, one between the two transverse directions $\hat{x} \sim \hat{y}$ and another between the two longitudinal (or "parallel") directions $\hat{z} \sim \hat{t}]$, the 24 correlation functions in Eq. (7) are grouped into eight equivalence classes, as shown in Table I. It must be noted that we can also group the eight correlation functions in Table I into three "overclasses" by the plaquette indices $\mu$ and $\nu$,

$$
\begin{aligned}
\mathcal{D}^{t t} & =\left\{\mathcal{D}_{\|}^{t t, t}, \mathcal{D}_{\perp}^{t t, p}\right\}, \\
\mathcal{D}^{t p} & =\left\{\mathcal{D}_{\|}^{t p, t}, \mathcal{D}_{\|}^{t p, p}, \mathcal{D}_{\perp}^{t p, t}, \mathcal{D}_{\perp}^{t p, p}\right\}, \\
\mathcal{D}^{p p} & =\left\{\mathcal{D}_{\perp}^{p p, t}, \mathcal{D}_{\|}^{p p, p}\right\} .
\end{aligned}
$$

In other words, the 24 correlation functions in Eq. (7) can be written, using the parametrization in Eq. (3), as linear combinations of eight linearly independent tensors $T^{(n)}$, e.g., the two fundamental tensors in Eq. (6) plus six other linearly independent tensors among those listed below Eq. (6), with eight (nonzero) independent functions $f_{n}$.
TABLE I. The eight equivalence classes of linearly independent correlation functions in which the 24 components of the correlator $\mathcal{D}_{\mu \nu, \xi}$, defined in Eq. (7), can be grouped. The superscripts $t$, $p$ stand respectively for the $\hat{x}, \hat{y}$ (transverse to $\vec{B}$ ) directions and for the $\hat{z}, \hat{t}$ (parallel to $\vec{B}$ ) directions.

\begin{tabular}{lc}
\hline \hline Class name & Elements $(\mu \nu, \xi)$ \\
\hline $\mathcal{D}_{\|}^{t t, t}$ & $(12,1),(12,2)$ \\
$\mathcal{D}_{\perp}^{t t, p}$ & $(12,3),(12,4)$ \\
$\mathcal{D}_{\|}^{t p, t}$ & $(13,1),(14,1),(23,2),(24,2)$ \\
$\mathcal{D}_{\|}^{t p, p}$ & $(13,3),(14,4),(23,3),(24,4)$ \\
$\mathcal{D}_{\perp}^{t p, t}$ & $(13,2),(14,2),(23,1),(24,1)$ \\
$\mathcal{D}_{\perp}^{t p, p}$ & $(13,4),(14,3),(23,4),(24,3)$ \\
$\mathcal{D}_{\perp}^{p p, t}$ & $(34,1),(34,2)$ \\
$\mathcal{D}_{\|}^{p p, p}$ & $(34,3),(34,4)$ \\
\hline \hline
\end{tabular}

[For these particular correlators, the contribution from any other possible tensor will be (i) simply zero, or (ii) a linear combination of the first eight independent tensors.] Now the question is the following: how should we parametrize the eight independent functions $f_{n}$, or, equivalently, their eight linear combinations which represent the eight functions listed in the first column of Table I? We use for these eight functions the following parametrization [which is a simple generalization of the parametrization (10) used in the $B=0$ case]:

$$
\begin{aligned}
\mathcal{D}_{\|}^{t t, t} & =\left[A_{0}^{t t}+A_{1}^{t t}\left(1-\frac{1}{2} \mu^{t t, t} d\right)\right] e^{-\mu^{t, t} d}+\frac{a_{\|}^{t t, t}}{d^{4}}, \\
\mathcal{D}_{\perp}^{t t, p} & =\left(A_{0}^{t t}+A_{1}^{t t}\right) e^{-\mu^{t, p} d}+\frac{a_{\perp}^{t t, p}}{d^{4}}, \\
\mathcal{D}_{\|}^{t p, t} & =\left[A_{0}^{t p}+A_{1}^{t p}\left(1-\frac{1}{2} \mu^{t p, t} d\right)\right] e^{-\mu^{t p, t} d}+\frac{a_{\|}^{t p, t}}{d^{4}}, \\
\mathcal{D}_{\|}^{t p, p} & =\left[\tilde{A}_{0}^{t p}+\tilde{A}_{1}^{t p}\left(1-\frac{1}{2} \mu^{t p, p} d\right)\right] e^{-\mu^{t p, p} d}+\frac{a_{\|}^{t p, p}}{d^{4}}, \\
\mathcal{D}_{\perp}^{t p, t} & =\left(A_{0}^{t p}+A_{1}^{t p}\right) e^{-\mu^{t, . t} d}+\frac{a_{\perp}^{t p, t}}{d^{4}}, \\
\mathcal{D}_{\perp}^{t p, p} & =\left(\tilde{A}_{0}^{t p}+\tilde{A}_{1}^{t p}\right) e^{-\mu^{t p, p} d}+\frac{a_{\perp}^{t p, p}}{d^{4}}, \\
\mathcal{D}_{\perp}^{p p, t} & =\left(A_{0}^{p p}+A_{1}^{p p}\right) e^{-\mu^{p, t} d}+\frac{a_{\perp}^{p p, t}}{d^{4}}, \\
\mathcal{D}_{\|}^{p p, p} & =\left[A_{0}^{p p}+A_{1}^{p p}\left(1-\frac{1}{2} \mu^{p p, p} d\right)\right] e^{-\mu^{p p, p} d}+\frac{a_{\|}^{p p, p}}{d^{4}},
\end{aligned}
$$

where the dependence of the various parameters on $B$ is understood and is discussed in the next section on the basis of the numerical results obtained by lattice simulations of $N_{f}=2$ QCD. Similar investigations exploring cases of 
broken Euclidean $S O(4)$ symmetry, like the finite-temperature case [50], show that the nonperturbative coefficients $A_{0}$ and $A_{1}$ are the quantities showing the most significant variation; however, in principle, both the perturbative and the nonperturbative coefficients, as well as the correlation length, might depend on the particular correlator class and on the value of the magnetic field. The only assumption that can and will be made a priori consists in the following constraint: $\tilde{A}_{0}^{t p}+\tilde{A}_{1}^{t p}=A_{0}^{t p}+A_{1}^{t p}$, meaning that, at $d=0$, the nonperturbative part of the correlation functions belonging to the same overclass, as defined by (12), has the same value. When $B=0$, the nonperturbative coefficients $A_{0}, A_{1}$ and $\mu$ no longer depend on the particular correlator class and, moreover, $\quad a_{\|}^{t t, t}=a_{\|}^{t p, t}=a_{\|}^{t p, p}=a_{\|}^{p p, p} \equiv a_{\|} \quad$ and $\quad a_{\perp}^{t t, p}=$ $a_{\perp}^{t p, t}=a_{\perp}^{t p, p}=a_{\perp}^{p p, t} \equiv a_{\perp}$, so that the eight functions in Eq. (13) reduce to the two functions $\mathcal{D}_{\|}$and $\mathcal{D}_{\perp}$ in Eq. (10), with $a_{\|} \equiv a_{0}-a_{1}$ and $a_{\perp} \equiv a_{0}+a_{1}$.

\section{NUMERICAL INVESTIGATION AND DISCUSSION}

We have considered $N_{f}=2$ QCD discretized via unimproved rooted staggered fermions and the standard plaquette action for the pure-gauge sector. The background magnetic field couples to the quark electric charges and its introduction corresponds to a modification of the Dirac operator: in the continuum an appropriate electromagnetic gauge field $A_{\mu}$ must be added to the covariant derivative, corresponding to additional $U(1)$ phases entering the elementary parallel transports in the discretized lattice version. For a magnetic field $\mathbf{B}=B \hat{\mathbf{z}}$ the functional integral reads

$$
\begin{aligned}
Z \equiv & \int \mathcal{D} U e^{-S_{G}} \operatorname{det} M^{\frac{1}{4}}\left[B, q_{u}\right] \operatorname{det} M^{\frac{1}{4}}\left[B, q_{d}\right], \\
M_{i, j}[B, q]= & a m \delta_{i, j}+\frac{1}{2} \sum_{\nu=1}^{4} \eta_{i, \nu}\left(u(B, q)_{i, \nu} U_{i, \nu} \delta_{i, j-\hat{\nu}}\right. \\
& \left.-u^{*}(B, q)_{i-\hat{\nu}, \nu} U_{i-\hat{\nu}, \nu}^{\dagger} \delta_{i, j+\hat{\nu}}\right),
\end{aligned}
$$

where $S_{G}$ is the gauge plaquette action, $q_{u}=2|e| / 3$ and $q_{d}=-|e| / 3(|e|$ being the elementary charge) are the quark electric charges, $i$ and $j$ refer to lattice sites and $\eta_{i, \nu}$ are the staggered phases.

The Abelian gauge field $A_{y}=B x$ and $A_{\mu}=0$ for $\mu=t$, $x, z$, which is a possible choice leading to $\mathbf{B}=B \hat{\mathbf{z}}$, is discretized on the lattice torus (we assume periodic boundary conditions in the spatial directions) as

$$
u_{i ; y}^{f}=e^{i a^{2} q_{f} B i_{x}},\left.\quad u_{i ; x}^{f}\right|_{i_{x}=L_{x}}=e^{-i a^{2} q_{f} L_{x} B i_{y}}
$$

with $u_{i ; \mu}^{f}=1$ elsewhere, while periodicity constraints impose to quantize $B$ as follows [52-54]:

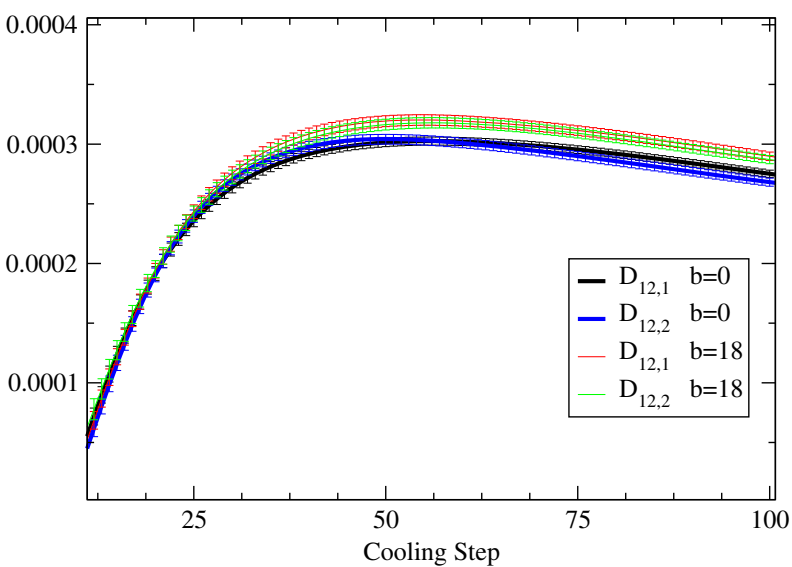

FIG. 1. Effect of cooling and of the magnetic field on the $\mathcal{D}_{12,1}$ and $\mathcal{D}_{12,2}$ correlation functions (evaluated for $d / a=8$ ), defined in Eq. (7). Both correlation functions belong to the $\mathcal{D}_{\|}^{t t, t}$ class, defined in Table I.

$$
|e| B=6 \pi b /\left(a^{2} L_{x} L_{y}\right) ; \quad b \in \mathbb{Z} .
$$

The correlator in Eq. (7) has been discretized through the following lattice observable [46]:

$$
\begin{aligned}
\mathcal{D}_{\mu \nu, \xi}^{L}(d)= & \left\langle\operatorname { T r } \left\{\Omega_{\mu \nu}^{\dagger}(x) S(x, x+d \hat{\xi})\right.\right. \\
& \left.\left.\times \Omega_{\mu \nu}(x+d \hat{\xi}) S^{\dagger}(x, x+d \hat{\xi})\right\}\right\rangle
\end{aligned}
$$

where $\Omega_{\mu \nu}(x)$ stands for the traceless anti-Hermitian part of the corresponding plaquette. In order to remove ultraviolet fluctuations, following previous studies of the gauge-field correlators, a cooling technique has been used [55,56] which, acting as a diffusion process, smooths out shortdistance fluctuations without touching physics at larger distances: for a correlator at a given distance $d$, this shows up as an approximate plateau in the dependence of the correlator on the number of cooling steps, whose location defines the value of the correlator. In Fig. 1, the effect of cooling on the correlation function is shown for one particular explored case: the value of the correlation function is taken at the maximum, and the error is assumed as the independent sum of a statistical error and systematic error due to the uncertainty in the determination of the plateau, estimated as the difference between the value at the maximum and the mean of the two neighboring points at the plateau.

We stress that the prescription adopted in the present study is one among other possible definitions of the correlators, which we have chosen consistently with previous lattice studies of the same quantities. It will be surely interesting, in the future, to investigate in more detail the issue of the dependence of the correlators on the smoothing procedure. Let us sketch the main open issues. First, one could adopt a different smoothing technique, like the so-called gradient flow [57]: while cooling and the 
gradient flow have been shown to provide equivalent results for the determination of topological quantities $[58,59]$, the situation could be different in the present case. Second, the adopted procedure implies that a different number of cooling steps is taken for different correlators; in particular, as noted in previous literature on the subject (see, e.g., Ref. [48]), the number of cooling steps at which the maximum is reached increases approximately quadratically with the correlator distance, as expected for a diffusion process. Therefore the adopted definition, consistently with previous studies, is one in which the regulator is scaled proportionally to the explored physical distance. Of course, one could adopt a different definition, in which the regulator (i.e. the number of cooling steps) is kept fixed: correlators at larger distances, for which the maximum is broader and resembles an extended plateau, and which are also the ones most sensitive to nonperturbative effects, do not change dramatically. In the present study, we regard such ambiguities as a possible systematic effect, related to the very definition of the correlator, which is not included in the reported errors. However, we note that a large part of this systematics is expected to cancel when one considers the dependence of the correlators on the magnetic field, which is the main issue considered in the present study. For that reason, a detailed comparison of different smoothing procedures is left to a forthcoming investigation.

Numerical simulations have been performed on a $24^{4}$ lattice by means of the rational hybrid Monte Carlo [60,61] algorithm implemented on GPU cards [62], with statistics of $O\left(10^{3}\right)$ molecular dynamics time units for each $b$ (with $b$ ranging from 0 to 27). The bare parameters have been set to $\beta=5.55$ and $a m=0.0125$, corresponding to a lattice spacing $a \simeq 0.125 \mathrm{fm}$ and to a pseudo-Goldstone pion mass $m_{\pi} \simeq 480 \mathrm{MeV}$ [63]. The correlators have been measured on about 100 configurations for each explored value of $|e| B$, chosen once every 20 molecular dynamics trajectories. The effects of autocorrelation in the data were assessed with a blocking procedure, and appear to be negligible.

\section{A. Results and analysis}

To give an example of the dependence of the correlators on the magnetic field, in Figs. 2-3 we plot results obtained respectively for the parallel and perpendicular classes, as a function of the distance, for the particular case $|e| B=1.46 \mathrm{GeV}^{2}$. Correlators are normalized to the values they take at zero external field. The huge error bars on the points at $d=3 a$ in Fig. 3 are due to the fact that, for the perpendicular correlators at that distance, the flat region surrounding the maximum is considerably narrower than for the other distances, and the procedure explained in the previous section to assess the systematic uncertainties takes this into account, yielding a larger error as one would reasonably expect. This happens both at $|e| B=0$ and

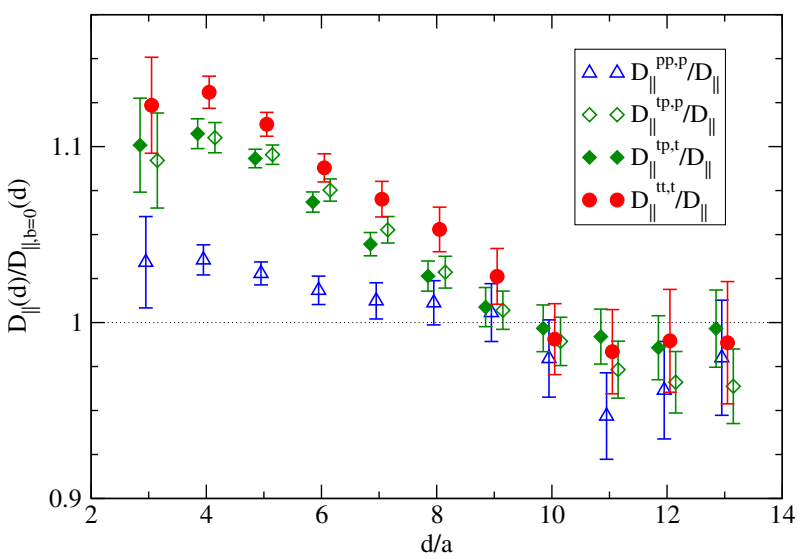

FIG. 2. Effect of the magnetic field on the parallel correlators: the ratio $\mathcal{D}_{\|}^{\text {class }}(d) / \mathcal{D}_{\|}(d)$ is plotted, where $\mathcal{D}_{\|}^{\text {class }}(d)$ is measured at $|e| B=1.46 \mathrm{GeV}^{2}$ and $\mathcal{D}_{\|}(d)$ at $B=0$. The data points are shifted horizontally for the sake of readability.

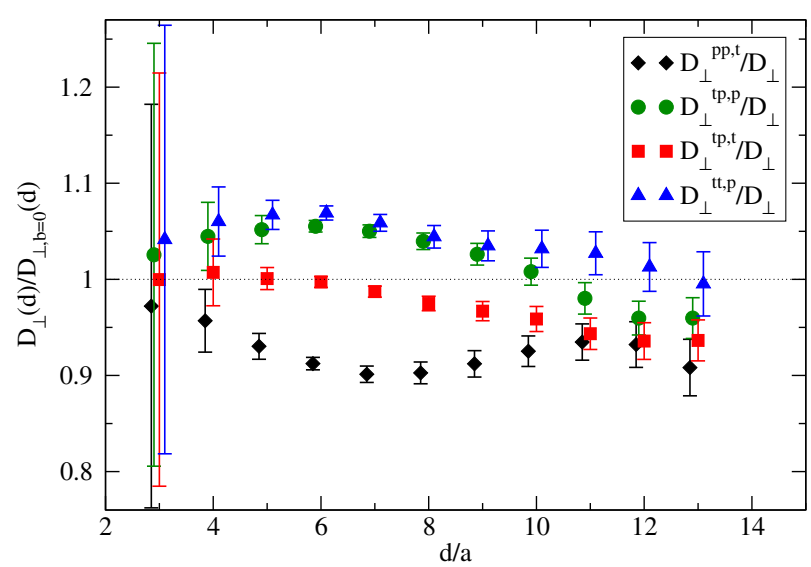

FIG. 3. Effect of the magnetic field on the perpendicular correlators: the ratio $\mathcal{D}_{\perp}^{\text {class }}(d) / \mathcal{D}_{\perp}(d)$ is plotted, where $\mathcal{D}_{\perp}^{\text {class }}(d)$ is measured at $|e| B=1.46 \mathrm{GeV}^{2}$ and $\mathcal{D}_{\perp}(d)$ at $|e| B=0$. The data points are shifted horizontally for the sake of readability.

$|e| B=1.46 \mathrm{GeV}^{2}$, and the resulting effect in the ratios plotted in Fig. 3 is even larger.

For each value of $|e| B$, we have fitted the correlators with the parametrization (13), including distances in the range $3 \leq d / a \leq 8$, thus obtaining an estimate for all parameters. From this first step, it has emerged that the eight parameters pertaining to the perturbative part of the correlation functions satisfy, within errors, the following equalities:

$$
\begin{aligned}
& a_{\|}^{t t, t} \simeq a_{\|}^{t p, t} \simeq a_{\|}^{t p, p} \simeq a_{\|}^{p p, p} \equiv a_{\|} \\
& a_{\perp}^{t t, p} \simeq a_{\perp}^{t p, t} \simeq a_{\perp}^{t p, p} \simeq a_{\perp}^{p p, t} \equiv a_{\perp},
\end{aligned}
$$

as it is possible to see from Table (II); moreover their dependence on $|e| B$ is negligible. This means that as far as the perturbative part of (13) is concerned, the parameters 
TABLE II. Values for the perturbative coefficients in Eq. (13), for $|e| B=1.46 \mathrm{GeV}^{2}$. The values of the corresponding parameters at $B=0$ are also reported for comparison.

\begin{tabular}{lcccc}
\hline \hline$a_{\|}(B=0)$ & $a_{\|}^{t t, t}$ & $a_{\|}^{t p, t}$ & $a_{\|}^{t p, p}$ & $a_{\|}^{p p, p}$ \\
$0.266(16)$ & $0.279(14)$ & $0.277(9)$ & $0.272(9)$ & $0.275(14)$ \\
$a_{\perp}(B=0)$ & $a_{\perp}^{t t, p}$ & $a_{\perp}^{t p, t}$ & $a_{\perp}^{t p, p}$ & $a_{\perp}^{p p, t}$ \\
$0.929(16)$ & $0.94(3)$ & $0.873(20)$ & $0.913(23)$ & $0.88(3)$ \\
\hline \hline
\end{tabular}

introduced in the $|e| B=0$ case are enough to describe our data.

Driven by this evidence, we have performed a best fit on all measured correlation functions for $b \leq 18$, assuming the perturbative parameters $a_{\perp}$ and $a_{\|}$to be independent of $|e| B$, but without making any further assumption about the $|e| B$-dependence of the other parameters. From this fit we obtain the satisfactory $\chi^{2} / n_{\text {dof }}=335 / 322$ : therefore the parametrization in Eq. (13) together with the assumptions in Eq. (19) are assumed in the following discussion.

In Fig. 4, the inverse of the correlation lengths is plotted as a function of $|e| B$. It is not trivial to give an interpretation of the data: they show a modest decrease for most of the correlation lengths, which amounts to about $5 \%-10 \%$ for the largest values of $|e| B$. We have also performed a fit setting the six correlation lengths in Eq. (13) equal; however in this case we have obtained $\chi^{2} / n_{\mathrm{dof}}=1237 / 358$, which is not satisfactory. We also notice that correlation lengths along the direction perpendicular to $B$ (empty symbols in Fig. 4) tend to be slightly smaller than the corresponding ones along the parallel direction.

We have also varied the fit range to assess the part of systematic error in our results which is related to this choice, exploring the ranges $3 \leq d / a \leq 7,4 \leq d / a \leq 8$ and $4 \leq d / a \leq 9$, in addition to $3 \leq d / a \leq 8$. The

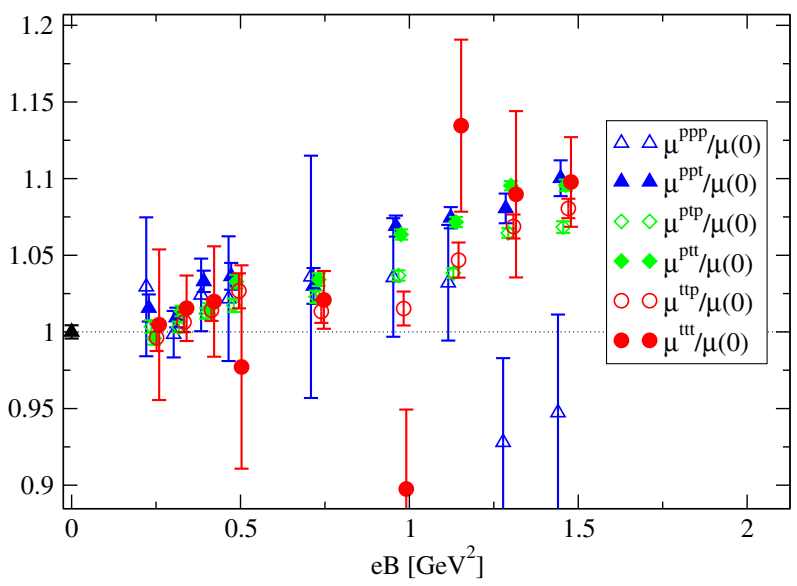

FIG. 4. Effect of the magnetic field on the (inverse) correlation lengths defined in parametrization (13): the ratio $\mu^{\text {class }}(|e| B) / \mu(0)$ is plotted. The value of the (inverse) correlation length for $|e| B=0$ is 0.721 (3) GeV. The data points are shifted horizontally for the sake of readability. estimates of the perturbative parameters $a_{\|}$and $a_{\perp}$ show a maximum variation around $10 \%$. The estimates of the correlation lengths have a maximum variation around 5\%; however, when the ratios to the corresponding values at $|e| B=0$ are considered (see Fig. 4), the systematic effect is comparable to or smaller in size than the statistical error.

Among the various parameters entering Eq. (13), the ones showing the most pronounced variation with $|e| B$ have been the nonperturbative coefficients $A_{0}$ and $A_{1}$. That implies a significant dependence on the magnetic field of the gluon condensate, which is discussed in detail in the next section.

\section{B. Gluon condensate}

The gluon condensate is defined as

$$
G_{2}=\frac{g^{2}}{4 \pi^{2}} \sum_{\mu \nu, a}\left\langle G_{\mu \nu}^{a} G_{\mu \nu}^{a}\right\rangle
$$

and is related to the correlator in Eq. (1) through an operator product expansion. It encodes the main effect of nonperturbative physics to gluon dynamics and its relevance was first pointed out in Ref. [64]. In the case of $|e| B \neq 0$ we can distinguish three contributions, coming from different sets of plaquettes in the sum in Eq. (20), in a fashion similar to Eq. (12):

$$
G_{2}=G_{2}^{t t}+G_{2}^{t p}+G_{2}^{p p} .
$$

For the relation between the gluon-field correlator (1) and $G_{2}$ the reader can refer to [48] and references therein; one obtains $G_{2}$ from the small distance limit of the nonperturbative contributions to the correlator; hence in practice, following the parametrization in Eq. (13), we obtain

$G_{2}(B)=\frac{1}{\pi^{2}}\left[A_{0}^{t t}+A_{1}^{t t}+4\left(A_{0}^{t p}+A_{1}^{t p}\right)+A_{0}^{p p}+A_{1}^{p p}\right]$.

In Fig. 5 we report the values obtained for $G_{2}$ as a function of $|e| B$, normalized to the value of the condensate obtained for $|e| B=0$, where we obtain $G_{2}=3.56(5) \times 10^{-2} \mathrm{GeV}^{4}$, the reported error being just the statistical one. We have also estimated the systematic error due to the choice of the fit range, as discussed in the previous subsection: the effect on the absolute value of $G_{2}$ at $|e| B=0$ is significant and amounts to $20 \%$ of the total value. We are therefore in rough agreement, taking also into account the unphysical mass spectrum of our discretization, with the phenomenological estimates of the gluon condensate [65], reporting $G_{2} \simeq 2.4(1.1) \times 10^{-2} \mathrm{GeV}^{4}$. Instead, when we consider the ratios of condensate to the $|e| B=0$ value, which are reported in Fig. 5, the systematic error turns out to be negligible as compared to the statistical one. 


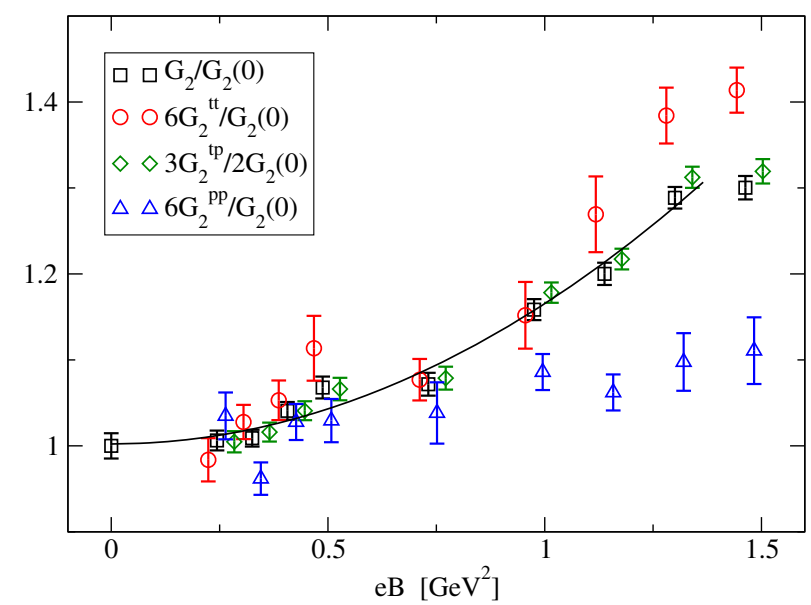

FIG. 5. Effect of the magnetic field on the gluon condensate $G_{2}$ obtained from the parametrization (13). The effects on the three different contributions are plotted along the total value. The data points are shifted horizontally for the sake of readability.

We notice that $G_{2}$ grows as a function of $|e| B$, the increase being of the order of $25 \%$ for the largest value of $|e| B$ explored. In the same figure we also report the relative increases in the $G_{2}^{t t}, G_{2}^{t p}$ and $G_{2}^{p p}$ terms. We see that the $t t$ term is the most affected by the magnetic field, whereas the $p p$ contribution shows a really modest dependence on $|e| B$. In Fig. 5, the best fit with a quadratic function

$$
\frac{G_{2}(|e| B)}{G_{2}(0)}=1+K(|e| B)^{2}
$$

is also plotted. We obtain $K=0.164(7) \mathrm{GeV}^{-4}$ and $\chi^{2} / n_{\mathrm{dof}}=1.52$, excluding the point at $|e| B=1.46 \mathrm{GeV}^{2}$. An increase of the chromomagnetic gluon condensate with $|e| B$ has also been found in [22], which is in qualitative agreement with the result presented here. A similar behavior for $G_{2}$ has also been predicted making use of QCD sum rules [66].

\section{CONCLUSIONS}

In this study we have explored the effects of a magnetic background field on the gauge-invariant two-point correlators of the gauge-field strength. Electromagnetic fields are coupled directly to quark fields; however, as for other puregauge observables, we did expect and we have indeed observed a nontrivial effect which can be interpreted in terms of nonperturbative quark-loop contributions. We have discussed the residual symmetries of the Lorentz group in the presence of a constant and uniform magnetic background, and how it affects the general structure of the correlators. We have then presented the numerical results of an exploratory lattice study performed for $N_{f}=2 \mathrm{QCD}$ discretized via rooted staggered fermions.
Our results can be summarized as follows. We have evidenced a significant effect of the magnetic field on the correlation functions (see Figs. 2-3). The short-distance, perturbative part of the correlators is practically unaffected by the presence of the magnetic background, while significant effects are observed for the nonperturbative part. In particular, one observes a mild effect on the nonperturbative correlation lengths, with a general tendency for a decrease of the correlation lengths as a function of $|e| B$, which is slightly more visible for correlators in the directions orthogonal to $|e| B$.

A larger effect, and a more significant anisotropy, is observed for the coefficients of the nonperturbative terms, which can be directly related to the gluon condensate [see Eq. (22)]. Due to the explicit Lorentz symmetry breaking caused by the magnetic field, we can distinguish among three different contributions to the gluon condensate. An analysis based on Eq. (22) shows that each term has a different behavior as a function of the magnetic field (see Fig. 5). Starting from that, we have observed that the gluon condensate itself increases as a function of $B$, with the increase being of the order of $20 \%$ for $|e| B \sim 1 \mathrm{GeV}^{2}$. Relative differences between the different contributions are of the same order of magnitude, meaning that anisotropies induced by $B$ are significant and comparable to those observed in other pure-gauge quantities (see, e.g., Ref. [35]). The increase of the gluon condensate provides evidence of the phenomenon known as gluon catalysis, which had been previously observed based on the magneticfield effects on plaquette expectation values [30,34,67]. We notice that the overall effect on the correlators, in particular, regarding the changes in the perturbative and nonperturbative parts, is similar to what has been observed in other setups where Lorentz symmetry is explicitly broken by external parameters, like for QCD at finite temperature [50].

In the future, we plan to repeat the present exploratory study by adopting a discretization of QCD at the physical point, i.e. with quark masses tuned at their phenomenological values, and by extending the investigation to other gauge-invariant correlation functions, like those involving quark fields [68]. It will be also interesting to study, within the stochastic vacuum model, which takes the correlators as an input, the effect of the magnetic background field on the static quark-antiquark potential, and in particular on the string tension, in order to obtain an indirect confirmation of the anisotropy of the potential which has been already observed by direct lattice measurements [35]. Finally, as we have already observed in Sec. III, in this work we have limited ourselves to the smoothing technique (cooling) adopted in previous lattice studies for the measure of the correlators, but one could also adopt the so-called gradient flow [57] as a regulator, and consider a different prescription for fixing the amount of smoothing: we postpone a careful comparison of different smoothing procedures for these particular observables to a forthcoming investigation. 


\section{ACKNOWLEDGMENTS}

F. N. acknowledges financial support from the INFN SUMA project. We thank the Scientific Computing Center at INFN Pisa and INFN Genoa for providing computing resources.

[1] D. Kharzeev, K. Landsteiner, A. Schmitt, and H. U. Yee, Strongly interacting matter in magnetic fields, Lect. Notes Phys. 871, 1 (2013).

[2] R. C. Duncan and C. Thompson, Formation of very strongly magnetized neutron stars-implications for gamma-ray bursts, Astrophys. J. 392, L9 (1992).

[3] V. Skokov, A. Y. Illarionov, and V. Toneev, Estimate of the magnetic field strength in heavy-ion collisions, Int. J. Mod. Phys. A 24, 5925 (2009).

[4] V. Voronyuk, V. D. Toneev, W. Cassing, E. L. Bratkovskaya, V. P. Konchakovski, and S. A. Voloshin, (Electro-)magnetic field evolution in relativistic heavy-ion collisions, Phys. Rev. C 83, 054911 (2011).

[5] A. Bzdak and V. Skokov, Event-by-event fluctuations of magnetic and electric fields in heavy-ion collisions, Phys. Lett. B 710, 171 (2012).

[6] W.-T. Deng and X.-G. Huang, Event-by-event generation of electromagnetic fields in heavy-ion collisions, Phys. Rev. C 85, 044907 (2012).

[7] K. Tuchin, Particle production in strong electromagnetic fields in relativistic heavy-ion collisions, Adv. High Energy Phys. 13 (2013) 490495.

[8] T. Vachaspati, Magnetic fields from cosmological phase transitions, Phys. Lett. B 265, 258 (1991).

[9] D. Grasso and H. R. Rubinstein, Magnetic fields in the early Universe, Phys. Rep. 348, 163 (2001).

[10] V. A. Miransky and I. A. Shovkovy, Magnetic catalysis and anisotropic confinement in QCD, Phys. Rev. D 66, 045006 (2002).

[11] M. N. Chernodub, Background magnetic field stabilizes QCD string against breaking, arXiv:1001.0570.

[12] M. M. Musakhanov and F. C. Khanna, The Axial anomaly and the conversion of gluons into photons, arXiv:hep-ph/ 9605232 .

[13] H. T. Elze and J. Rafelski, in Sandansky 1998, Frontier Tests of QED and Physics of the Vacuum, edited by E. Zavattini, D. Bakalov, and C. Rizzo (Heron Press, Sofia, 1998), p. 447 pages.

[14] H. T. Elze, B. Muller, and J. Rafelski, Interfering QCD/QED vacuum polarization, hep-ph/9811372.

[15] M. Asakawa, A. Majumder, and B. Muller, Electric charge separation in strong transient magnetic fields, Phys. Rev. C 81, 064912 (2010).

[16] B. V. Galilo and S. N. Nedelko, Impact of the strong electromagnetic field on the QCD effective potential for homogeneous Abelian gluon-field configurations, Phys. Rev. D 84, 094017 (2011).

[17] M. A. Andreichikov, V. D. Orlovsky, and Y. A. Simonov, Asymptotic Freedom in Strong Magnetic Fields, Phys. Rev. Lett. 110, 162002 (2013).
[18] T. Kojo and N. Su, The quark mass gap in a magnetic field, Phys. Lett. B 720, 192 (2013).

[19] T. Kojo and N. Su, A renormalization group approach for QCD in a strong magnetic field, Phys. Lett. B 726, 839 (2013).

[20] P. Watson and H. Reinhardt, Quark gap equation in an external magnetic field, Phys. Rev. D 89, 045008 (2014).

[21] J. O. Andersen, W. R. Naylor, and A. Tranberg, Chiral and deconfinement transitions in a magnetic background using the functional renormalization group with the Polyakov loop, J. High Energy Phys. 04 (2014) 187.

[22] S. Ozaki, QCD effective potential with strong U(1) em magnetic fields, Phys. Rev. D 89, 054022 (2014).

[23] K. Kamikado and T. Kanazawa, Chiral dynamics in a magnetic field from the functional renormalization group, J. High Energy Phys. 03 (2014) 009.

[24] N. Mueller, J. A. Bonnet, and C. S. Fischer, Dynamical quark mass generation in a strong external magnetic field, Phys. Rev. D 89, 094023 (2014).

[25] Y. A. Simonov and M. A. Trusov, Confinement and $\alpha_{s}$ in a strong magnetic field, Phys. Lett. B 747, 48 (2015).

[26] G. Cao and X. G. Huang, Electromagnetic triangle anomaly and neutral pion condensation in QCD vacuum, arXiv:1509.06222.

[27] M. D'Elia, S. Mukherjee, and F. Sanfilippo, QCD phase transition in a strong magnetic background, Phys. Rev. D 82, 051501 (2010).

[28] M. D'Elia and F. Negro, Chiral properties of strong interactions in a magnetic background, Phys. Rev. D 83, 114028 (2011).

[29] G. S. Bali, F. Bruckmann, G. Endrodi, Z. Fodor, S. D. Katz, S. Krieg, A. Schafer, and K. K. Szabo, The QCD phase diagram for external magnetic fields, J. High Energy Phys. 02 (2012) 044.

[30] E.-M. Ilgenfritz, M. Kalinowski, M. Muller-Preussker, B. Petersson, and A. Schreiber, Two-color QCD with staggered fermions at finite temperature under the influence of a magnetic field, Phys. Rev. D 85, 114504 (2012).

[31] G. S. Bali, F. Bruckmann, G. Endrodi, F. Gruber, and A. Schaefer, Magnetic field-induced gluonic (inverse) catalysis and pressure (an)isotropy in QCD, J. High Energy Phys. 04 (2013) 130.

[32] M. D'Elia, M. Mariti, and F. Negro, Susceptibility of the QCD Vacuum to CP-Odd Electromagnetic Background Fields, Phys. Rev. Lett. 110, 082002 (2013).

[33] F. Bruckmann, G. Endrodi, and T. G. Kovacs, Inverse magnetic catalysis and the Polyakov loop, J. High Energy Phys. 04 (2013) 112.

[34] E.-M. Ilgenfritz, M. Muller-Preussker, B. Petersson, and A. Schreiber, Magnetic catalysis (and inverse catalysis) at finite 
temperature in two-color lattice QCD, Phys. Rev. D 89, 054512 (2014).

[35] C. Bonati, M. D'Elia, M. Mariti, M. Mesiti, F. Negro, and F. Sanfilippo, Anisotropy of the quark-antiquark potential in a magnetic field, Phys. Rev. D 89, 114502 (2014).

[36] C. Bonati, M. D'Elia, and A. Rucci, Heavy quarkonia in strong magnetic fields, Phys. Rev. D 92, 054014 (2015).

[37] A. Di Giacomo, H. G. Dosch, V. I. Shevchenko, and Y. A. Simonov, Field correlators in QCD: Theory and applications, Phys. Rep. C 372, 319 (2002).

[38] D. Gromes, Space-time dependence of the gluon condensate correlation function and quarkonium spectra, Phys. Lett. 115B, 482 (1982).

[39] M. Campostrini, A. Di Giacomo, and G. Mussardo, Correlation length of the vacuum condensate in lattice gauge theories, Z. Phys. C 25, 173 (1984).

[40] M. Campostrini, A. Di Giacomo, and S. Olejnik, On the possibility of detecting gluon condensation from the spectra of heavy quarkonia, Z. Phys. C 31, 577 (1986).

[41] Y. A. Simonov, S. Titard, and F. J. Yndurain, Heavy quarkonium systems and nonperturbative field correlators, Phys. Lett. B 354, 435 (1995).

[42] H. G. Dosch, Gluon condensate and effective linear potential, Phys. Lett. B 190, 177 (1987).

[43] H. G. Dosch and Y. A. Simonov, The area law of the Wilson loop and vacuum field correlators, Phys. Lett. B 205, 339 (1988).

[44] Y. A. Simonov, Vacuum background fields in QCD as a source of confinement, Nucl. Phys. B307, 512 (1988).

[45] Y. A. Simonov, Pion decay constants in a strong magnetic field, arXiv:1503.06616.

[46] A. Di Giacomo and H. Panagopoulos, Field strength correlations in the QCD vacuum, Phys. Lett. B 285, 133 (1992).

[47] A. Di Giacomo, E. Meggiolaro, and H. Panagopoulos, Gauge-invariant field strength correlations in QCD at zero and non-zero temperature, Nucl. Phys. B483, 371 (1997).

[48] M. D'Elia, A. Di Giacomo, and E. Meggiolaro, Field strength correlators in full QCD, Phys. Lett. B 408, 315 (1997).

[49] E. Meggiolaro, Field strength correlators in QCD: new fits to the lattice data, Phys. Lett. B 451, 414 (1999).

[50] M. D'Elia, A. Di Giacomo, and E. Meggiolaro, Gaugeinvariant field-strength correlators in pure Yang-Mills theory and full QCD at finite temperature, Phys. Rev. D 67, 114504 (2003).

[51] G. Bali, N. Brambilla, and A. Vairo, A lattice determination of QCD field strength correlators, Phys. Lett. B 421, 265 (1998).

[52] G. 't Hooft, A property of electric and magnetic flux in nonAbelian gauge theories, Nucl. Phys. B153, 141 (1979).

[53] P. H. Damgaard and U. M. Heller, The U(1) Higgs model in an external electromagnetic field, Nucl. Phys. B309, 625 (1988).

[54] M. H. Al-Hashimi and U. J. Wiese, Discrete accidental symmetry for a particle in a constant magnetic field on a torus, Ann. Phys. (Amsterdam) 324, 343 (2009).

[55] M. Campostrini, A. Di Giacomo, M. Maggiore, H. Panagopoulos, and E. Vicari, Cooling and the string tension in lattice gauge theories, Phys. Lett. B 225, 403 (1989); A. Di Giacomo, M. Maggiore, and S. Olejnik, Evidence for flux tubes from cooled QCD configurations, Phys. Lett. B 236, 199 (1990).

[56] B. Berg, Dislocations and topological background in the lattice $\mathrm{O}(3) \sigma$ model, Phys. Lett. 104B, 475 (1981); Y. Iwasaki and T. Yoshie, Instantons and topological charge in lattice gauge theory, Phys. Lett. 131B, 159 (1983); S. Itoh, Y. Iwasaki, and T. Yoshie, Stability of instantons on the lattice and the renormalized trajectory, Phys. Lett. 147B, 141 (1984); M. Teper, Instantons in the quantized SU(2) vacuum: a lattice Monte Carlo investigation, Phys. Lett. 162B, 357 (1985); E. M. Ilgenfritz, M. L. Laursen, G. Schierholz, M. Muller-Preussker, and H. Schiller, First evidence for the existence of instantons in the quantized SU(2) lattice vacuum, Nucl. Phys. B268, 693 (1986).

[57] M. Lüscher, Trivializing maps, the Wilson flow and the HMC algorithm, Commun. Math. Phys. 293, 899 (2010); Properties and uses of the Wilson flow in lattice QCD, J. High Energy Phys. 08 (2010) 071; J. High Energy Phys. 03 (2014) 092.

[58] C. Bonati and M. D'Elia, Comparison of the gradient flow with cooling in $S U(3)$ pure gauge theory, Phys. Rev. D 89, 105005 (2014).

[59] C. Alexandrou, A. Athenodorou, and K. Jansen, Topological charge using cooling and the gradient flow, Phys. Rev. D 92, 125014 (2015).

[60] S. A. Gottlieb, W. Liu, D. Toussaint, R. L. Renken, and R. L. Sugar, Hybrid molecular dynamics algorithms for the numerical simulation of quantum chromodynamics, Phys. Rev. D 35, 2531 (1987).

[61] A. D. Kennedy, I. Horvath, and S. Sint, A new exact method for dynamical fermion computations with nonlocal actions, Nucl. Phys. B, Proc. Suppl. 73, 834 (1999).

[62] C. Bonati, G. Cossu, M. D'Elia, and P. Incardona, QCD simulations with staggered fermions on GPUs, Comput. Phys. Commun. 183, 853 (2012).

[63] S. Gottlieb, A. Krasnitz, U. M. Heller, A. D. Kennedy, J. B. Kogut, R. L. Renken, D. K. Sinclair, R. L. Sugar, D. Toussaint, and K.C. Wang, Thermodynamics of lattice QCD with two light quarks on a $16^{3} \times 8$ lattice, Phys. Rev. D 47, 3619 (1993).

[64] M. A. Shifman, A. I. Vainshtein, and V. I. Zakharov, QCD and resonance physics. theoretical foundations, Nucl. Phys. B147, 385 (1979).

[65] S. Narison, Heavy quarkonia mass splittings in QCD: gluon condensate, alpha-s and 1/m expansion, Phys. Lett. B 387, 162 (1996).

[66] A. Ayala, C. A. Dominguez, L. A. Hernandez, M. Loewe, J. C. Rojas, and C. Villavicencio, Quark deconfinement and gluon condensate in a weak magnetic field from QCD sum rules, Phys. Rev. D 92, 016006 (2015).

[67] G. S. Bali, F. Bruckmann, G. Endrodi, F. Gruber, and A. Schaefer, Magnetic field-induced gluonic (inverse) catalysis and pressure (an)isotropy in QCD, J. High Energy Phys. 04 (2013) 130.

[68] M. D’Elia, A. Di Giacomo, and E. Meggiolaro, Gaugeinvariant quark-antiquark nonlocal condensates in lattice QCD, Phys. Rev. D 59, 054503 (1999). 